\title{
TTR
}

Traduction, terminologie, re?daction

\section{Sur la thèse quinéenne de l'indétermination de la traduction}

\section{Vera Vidal}

Volume 3, numéro 1, 1er semestre 1990

L'agora de la traduction

URI : https://id.erudit.org/iderudit/037057ar

DOI : https://doi.org/10.7202/037057ar

Aller au sommaire du numéro

Éditeur(s)

Association canadienne de traductologie

ISSN

0835-8443 (imprimé)

1708-2188 (numérique)

Découvrir la revue

Citer cet article

Vidal, V. (1990). Sur la thèse quinéenne de l'indétermination de la traduction. TTR, 3(1), 31-48. https://doi.org/10.7202/037057ar

Tous droits réservés (C TTR: traduction, terminologie, rédaction — Les auteurs, 1990
Ce document est protégé par la loi sur le droit d'auteur. L’utilisation des services d'Érudit (y compris la reproduction) est assujettie à sa politique d'utilisation que vous pouvez consulter en ligne.

https://apropos.erudit.org/fr/usagers/politique-dutilisation/ 


\section{Sur la thèse quinéenne de l'indétermination de la traduction}

\section{Vera Vidal}

Si l'importance d'un philosophe se mesure à l'ampleur des querelles que ses thèses suscitent, W.V.O. Quine mérite d'être considéré comme l'un des plus grands penseurs du $\mathrm{XX}^{\circ}$ siècle. Il a su poser des questions et formuler des thèses en philosophie analytique du langage qui n'ont laissé indifférent aucun chercheur dans ce domaine.

Parmi les thèses les plus débattues, celle de l'indétermination de la traduction a déclenché une interminable série de controverses dès sa formulation dans Word and Object en 1960.

M. Friedman soutient qu'il s'agit de «la thèse la plus connue, qui a soulevé le plus grand nombre de discussions dans toute la littérature philosophique contemporaine». ${ }^{1}$ Nous préférons, quant à nous, dire qu'elle reste incomprise et mal interprétée.

Quine est un philosophe dont on parle beaucoup, mais que l'on lit peu et mal. Du moins, c'est l'impression qui se dégage de la plupart des critiques qui lui sont adressées: elles reflètent une profonde méconnaissance de l'ensemble de la pensée quinéenne.

La philosophie de Quine présente un aspect systématique: si l'on détache ses thèses de l'ensemble, elles peuvent perdre leur signification, ce qui conduit à de mauvaises interprétations et à des critiques mal fondées.

L'objectif de cet essai est donc de présenter la thèse quinéenne de l'indétermination de la traduction, en essayant d'expli-

1. Friedman, M. (1975). «Physicalism and the Indeterminacy of Translation», Nous, p. 353. 
quer son émergence dans l'ensemble systématique de sa philosophie; en d'autres termes, de saisir, à travers ses relations avec les autres thèses, ce qui la rend significative et renforce l'argumentation qui la justifie.

\section{Le projet épistémologique de Quine}

Notre lecture de Quine nous a conduit à penser que l'ensemble de sa philosophie est une réponse à ce que nous appelons son projet épistémologique, lequel apparaît clairement formulé dès la première page de Roots of Reference:

Given only the evidence of our senses, how do we arrive at our theory of the world?

Par «theory of the world», il entend la totalité de nos visions du monde, y compris celle du sens commun, ainsi que les visions scientifiques et philosophiques, lesquelles ne se distinguent du sens commun que par leurs degrés de complexité linguistique et analytique.

Selon lui, aucune vision du monde n'est naïve, ni capable de refléter la réalité, à l'instar d'un bon miroir. Elle est toujours le produit de conjectures plus ou moins sophistiquées que les hommes ont «inventées» à travers l'histoire pour leur permettre de s'expliquer, eux-mêmes et tout ce qui les entoure. Ces conjectures dépassent infiniment les informations fournies par les données de notre sensibilité - le seul critère d'《évidence» vraiment fiable pour Quine.

En dépit du fait que ce projet épistémologique n'a été clairement formulé que dans un ouvrage publié en 1974, on se rend compte qu'il a toujours été le principe moteur de ses investigations philosophiques.

La question fondamentale de Quine, celle que nous citons ci-dessus, repose sur deux présupposés fondamentaux de sa philosophie:

- la conviction que le seul critère d'évidence que l'on ne peut mettre en cause est l'évidence empirique;

- la constatation que nous avons tous une théorie du monde.

Attendu que, si nous déduisions toutes nos croyances des informations fournies par l'évidence sensible, nous n'arriverions jamais à justifier la totalité de notre savoir puisqu'il dépasse énormément ces seules données, le problème qui se pose aux épistémologues sera de justifier les rapports entre notre discours et la réalité, entre les mots et les objets.

Ce projet épistémologique se révèle par conséquent comme un effort pour répondre à la question de savoir comment lier les 
excitations de nos surfaces sensibles par les stimuli physiques venus du monde extérieur à la production d'une théorie du monde.

Il faut ajouter que, selon Quine, une théorie est un ensemble d'énoncés ordonnés par des règles très strictes, faisant partie d'un système linguistique qui leur accorde signification et degré de vérité. Tout ce que nous connaissons du monde extérieur ou intérieur est compris dans une vaste et complexe structure verbale. Ainsi, notre connaissance se révèle à travers le discours; elle est imbriquée dans la structure du langage. Ce qui fait de nous des «prisonniers» du discours.

On comprend ainsi pourquoi Quine choisit d'aborder la question épistémologique qui l'intéresse par le biais de l'analyse du langage: il croit au parallélisme langage-théorie, et considère toutes les théories comme des sous-ensembles du langage.

Nous nous servons d'une structure verbale très étendue d'énoncés qui sont liés les uns aux autres de diverses façons. Mais cette structure constitue une unité - et c'est cet ensemble qui comprend tout ce que nous sommes capables de connaître du monde. ${ }^{2}$

Le choix quinéen de suivre la méthode d'analyse linguistique n'exclut pas le monde extra-linguistique que le discours envisage de décrire.

Toujours fidèle à ses postulats empiriques, Quine est convaincu que toute analyse du langage doit suivre une rigoureuse méthode d'observation du comportement des locuteurs, de la relation du discours avec les stimuli physiques dont il dérive toujours, par des processus plus ou moins évidents. Bref, on doit suivre en épistémologie et en linguistique la méthode de travail des sciences de la nature. Ce faisant, Quine répudie toutes les analyses qui font appel à des entités mentales ou non observables.

L'orientation de son projet épistémologique conduit Quine à tenter de comprendre et de justifier la transcendance du discours par rapport à l'évidence des données sensorielles; il choisit pour mener cette analyse deux situations:

- celle de l'enfant qui apprend sa langue maternelle;

- celle d'un linguiste qui cherche à comprendre le langage d'un peuple dont il ne connaît ni la langue ni même la culture situation de traduction radicale.

De l'analyse de ces deux cas dérivera la totalité de ses propositions dans le domaine de la philosophie du langage et aussi

2. Quine, W.V.O. Word and Object (Cambridge, M.I.T. Press, 1960), p. 12. 
dans ceux de l'épistémologie, de l'ontologie et de la philosophie de la logique.

Notre interprétation de Quine ne suit pas l'ordre chronologique de la présentation de ses thèses. Nous croyons, par exemple, que le fait que sa théorie de l'apprentissage du langage chez l'enfant ne soit pas clairement présentée dans ses premiers articles philosophiques n'empêche pas qu'elle ait toujours été à la base de ses argumentations.

Notre lecture tente de restituer le sens épistémologique de ses investigations. Elle s'efforce d'intégrer ses thèses dans la totalité de son système. Nous croyons que c'est la meilleure façon de rendre compte de la rigueur de sa pensée et d'éviter des interprétations mal fondées.

\section{La théorie quinéenne du langage}

Elle est au centre, voire à la base, du système quinéen. Deux options fondamentales dominent son analyse du langage:

- le naturalisme, qui se révèle dans la conviction que les théories du langage doivent être soumises à la vérification empirique, puisqu'elles s'occupent de phénomènes naturels et observables: le discours, les actes du langage, les réponses que ces actes provoquent chez ceux qui les comprennent, sans négliger le processus d'acquisition du langage;

- le behaviorisme, selon lequel toute analyse linguistique doit s'appuyer sur l'observation du comportement des locuteurs et éviter le recours à des interprétations mentalistes. «En Psychologie, on peut choisir d'être ou de ne pas être behavioriste, mais en linguistique on n'a pas le choix. $n^{3}$

Cette conviction dérive de l'aspect social du processus d'apprentissage du langage: «Le langage est un art social qu'on acquiert par l'observation du comportement des locuteurs et dans des circonstances publiquement observables. ${ }^{4}$

On verra dans la section que nous consacrons à l'apprentissage du langage que le mécanisme behavioriste stimulus-réponse joue un rôle décisif dans la théorie quinéenne: on apprend à parler par un processus qui commence comme une réponse à des stimuli physiques et verbaux. De plus, Quine croit que la totalité de notre

3. Quine, W.V.O. «Indeterminacy of Translation Again», The Journal of Philosophy, vol. LXXXIV, nº 1, 1987, p. 5.

4. Quine, W.V.O. Ontological Relativity and Other Essays (New York, Columbia U.P., 1969), p. 168. 
discours est une réponse élaborée à partir des stimulations physiques du monde extérieur sur nos terminaisons nerveuses.

À noter que, contrairement à ce qu'affirment nombre de ses critiques, Quine reconnaît les insuffisances de la méthode behavioriste pour rendre compte de la complexité du phénomène linguistique, mais il s'insurge contre l'idée de la remplacer par des analyses faisant appel à des entités mentales ou abstraites.

Son projet de naturalisation de l'épistémologie l'amène à chercher des explications rigoureuses pour l'analyse du processus du langage et à les attendre du côté des sciences de la nature, de la neurologie, plutôt que des théories mentalistes. Nous croyons que beaucoup de ce que Quine envisageait - à la fois à titre de méthode et de résultats - se rapproche du travail développé aujourd'hui dans le champ des sciences cognitives.

\section{La théorie de l'apprentissage du langage}

Cette théorie occupe, elle aussi, une place décisive et joue un rôle-clé pour la bonne compréhension des thèses de Quine.

De l'analyse du processus d'apprentissage du langage maternel chez l'enfant, Quine en vient à constater que l'enfant commence par établir une étroite liaison avec les données sensibles; les premiers mots, appris sont ceux qui se réfèrent aux objets physiques. C'est dans une étape dérivée et plus complexe qu'on apprend les mots qui s'appliquent aux sensations, aux entités abstraites, aux ensembles, aux relations, aux entités mathématiques. De plus, les énoncés d'observation sont les premiers qu'un enfant prononce quand il apprend à parler. suivantes:

Selon lui, l'apprentissage du langage traverse les étapes

\section{$1^{\text {ère }}$ étape: Réponse aux stimuli physiques et verbaux}

Au début du processus, les enfants reçoivent des stimuli des objets qui touchent leurs terminaisons nerveuses, en même temps qu'ils reçoivent une stimulation verbale. La répétition de cet ensemble (stimulation physique et verbale dans le cadre de quelques situations semblables) leur permet de commencer à employer correctement les tournures de la langue.

Quine reconnaît que ce mécanisme behavioriste serait insuffisant pour justifier la totalité du processus. Ainsi, il n'arrive pas à justifier le fait qu'un enfant, d'après quelques situations concrètes d'apprentissage, soit capable de généraliser et d'utiliser telles expressions dans toutes les situations adéquates possibles. 
C'est pourquoi il introduit la notion d'«espace qualitatif inné». Il s'agit d'une norme de similarité qui nous permet de généraliser et d'acquérir des habitudes, sans laquelle tous les stimuli seraient tout à fait semblables. Si cette norme est fondamentale pour tous les processus d'apprentissage, elle ne peut pas être apprise; elle serait donc innée.

Avant d'être accusé de développer un argument anti-empiriste, il se défend en disant qu'il s'agit d'une propriété assez connue dans les études de psychologie du comportement, et qu'on peut l'explorer et l'observer en laboratoire. Les animaux sont régis, eux aussi, par cette norme. Elle est à la base de tous les processus inductifs, de nos généralisations et de nos attentes face à l'avenir.

Puisqu'il s'agit d'une capacité commune à tous les locuteurs, elle garantit une acceptabilité assez générale des inductions individuelles.

Un critère de décision qui permet à l'enfant de savoir s'il a utilisé l'expression adéquate à la situation dans laquelle il l'a employée est l'approbation ou l'incompréhension de son locuteur. C'est le dialogue qui permet de constater si l'apprenti a réussi ou échoué. L'approbation l'amènera à répéter ce comportement dans des situations semblables; l'incompréhension l'amènera à l'éviter. En somme, c'est par un processus behavioriste de réussite ou d'échec dans son dialogue que l'apprenti progresse.

Partant de cette analyse, Quine élabore la notion de signification-stimulus: ce sont des paires ordonnées de classes de stimulation, capables de provoquer une réponse positive ou négative du sujet devant l'émission de cette expression dans des situations concrètes.

La signification-stimulus n'est assimilable ni à la stimulation verbale, ni à la stimulation physique en tant que telles, mais à leur conjugaison avec une certaine situation, ce qui provoque une réponse positive ou négative du sujet qui se trouve dans cette situation.

\section{$2^{e}$ étape: La substitution par analogie}

A ce stade, l'enfant est capable d'effectuer des substitutions dans les structures déjà connues. Ainsi, à partir de "Voilà mon bras" et «ma main», il peut dire: "Voilà ma main». Les substitutions sont faites d'abord dans les énoncés d'observation et, plus tard, dans les énoncés abstraits.

Quine est convaincu que ces deux étapes du processus d'acquisition du langage expliquent une partie très restreinte de la totalité de notre habileté linguistique. 
D'autres associations inter-verbales sont nécessaires; elles rendent possible l'usage de nouveaux énoncés sans qu'ils soient attachés même secondairement à un quelconque domaine fixe de stimulations verbales. ${ }^{5}$

Apprendre par ostension c'est apprendre par simple induction et le mécanisme d'un tel apprentissage est le conditionnement. Mais cette méthode est incapable de nous conduire très loin dans le langage. ${ }^{6}$

\section{$3^{e}$ étape: L'inter-animation des énoncés}

Même si l'origine de notre discours a été une réponse aux stimulations physiques, et si le processus d'apprentissage linguistique a, lui aussi, commencé par être une réponse à de telles stimulations, c'est seulement quand on dépasse la phase behavioriste qu'on progresse effectivement. Dans cette troisième étape, le discours s'éloigne de la stimulation directe venue des objets physiques.

A ce stade du processus, les énoncés interagissent. Cela veut dire que même les énoncés d'observation peuvent ne plus être la réponse immédiate à une stimulation physique. Leur production dépend dès lors d'un réseau d'énoncés, quelquefois très complexe, réseau qui leur apporte à la fois signification, référence et valeur de vérité. Par exemple, quand un chercheur, dans un laboratoire de chimie, réalise une série de mélanges de produits et observe que la substance résultante est bleue, il prononce l'énoncé d'observation: «il y a du cuivre». Il s'agit d'un énoncé d'observation, prononcé en réponse à une stimulation physique, mais sa signification et sa valeur de vérité proviennent de l'ensemble de la théorie chimique dont l'énoncé fait partie. Quelqu'un qui ne connaîtrait pas la théorie ne serait pas en mesure de prononcer un tel énoncé en présence de cette stimulation physique.

On voit donc que la plus grande partie du réseau linguistique est constituée d'énoncés qui sont des réponses à d'autres énoncés, et que leur cohérence dérive de leurs rapports réciproques à l'intérieur de la théorie à laquelle ils appartiennent. Ils dépendent des tègles logiques, des lois causales, des présupposés et principes internes des théories et, enfin, de composantes culturelles.

La force du réseau théorique est telle qu'on ne peut plus retrouver dans le complexe le simple dont il est issu et qu'on n'est

5. Quine, W.V.O. Word and Object (Cambridge, M.I.T. Press, 1960), p. 10.

6. Quine, W.V.O. in Mind and Language, S. Guttenplan, ed. (Oxford, Clarendon, 1975), p. 10. 
plus capable d'identifier l'énoncé d'observation qui a été à l'origine du processus ayant abouti à un certain énoncé théorique.

La question de savoir dans quel cas un énoncé a été appris en réponse directe aux stimulations physiques et dans quel cas il a été appris dans le cadre d'un processus d'inter-animation appartient à l'histoire déjà oubliée (et irrécupérable) de chaque locuteur. Selon Quine, on est ainsi en mesure de localiser, dans le procès même d'inter-animation, le moment où le discours dépasse la phase behavioriste initiale pour entrer dans un processus de production de nouveaux énoncés à partir de l'ensemble qui constitue la théorie du monde de chaque individu à chaque époque.

La priorité qu'acquiert le linguistique sur le non-linguistique entraîne l'affaiblissement progressif du contenu sensoriel du discours encore prépondérant en début de processus. La connaissance indirecte prédomine. En effet, le locuteur se trouve entouré d'instruments linguistiques, d'un ensemble de règles qui favorisent l'homogénéisation de ses perceptions individuelles en vue d'un possible accord qui permette le dialogue. Autrement dit, le locuteur parlera selon le modèle linguistique et culturel de sa communauté.

Le discours devient incapable d'être le miroir de la réalité et d'atteindre une neutralité ou une objectivité véritable. Le processus d'apprentissage du langage exige qu'on utilise des structures linguistiques, référentielles, logiques et, enfin, culturelles. Ce qui dépasse largement le cadre des réponses aux stimulations physiques qui ont déclenché le processus. Des conventions, des «inventions» complètent les lacunes que créent les données d'observation. Il est impossible de distinguer dans le discours ce qui est objectif de ce qui est «production humaine»; ce qui est «substantiel» de ce qui est «style».

Par cette analyse, Quine réalise son projet épistémologique: on est désormais en mesure de comprendre comment les stimulations de nos terminaisons nerveuses se lient à notre théorie du monde. De comprendre aussi pourquoi cette théorie se compose d'énoncés qui affirment beaucoup plus que ce qu'on aurait pu affirmer si on s'était limité à produire des énoncés qui ne seraient que des réponses aux stimulations physiques immédiates.

\section{Le mythe de la signification}

L'analyse du processus d'apprentissage du langage révèle à Quine que l'observation du comportement des locuteurs ne permet aucune réponse à la question: qu'est-ce que la signification d'une expression linguistique?

Apprendre à utiliser correctement une expression veut dire qu'on a compris sa signification. Il est à remarquer qu'on n'ap- 
prend rien sur la nature de la signification: s'agit-il d'une entité? de quelle sorte? comment peut-on être sûr que deux expressions ont (ou n'ont pas) la même signification?

Par fidélité à sa méthode behavioriste et à son approche naturaliste du langage, Quine se rebelle contre le recours à la notion de signification. Il reconnaît que, si les mots servent à la communication, c'est parce qu'ils signifient, mais il refuse de postuler l'existence de la signification comme une entité sous-jacente à toutes les expressions linguistiques. Une telle supposition conduit à ce qu'il appelle «le mythe de la signification»:

On se figure qu'il existe une sorte de musée, où les idées sont exposées comme des tableaux et que chaque idée est accrochée à l'expression qu'elle signifie; chaque proposition serait notamment accrochée à un énoncé approprié. ${ }^{7}$

Si l'on croit à ce mythe, on finit par attribuer une signification à chaque signe linguistique, ce qui est semblable au processus par lequel on attribue un nom propre à chaque cuvre d'art. Ainsi, la signification devient une entité nommée par les signes linguistiques.

Sa critique de la réification de la signification n'est pas la conséquence d'une tendance nominaliste d'économie ontologique ou d'un refus des entités abstraites. Quine attaque cette notion parce qu'on en peut déduire les notions selon lui fallacieuses de synonymie, de proposition, d'analyticité, etc. Cet ensemble de notions mal définies nous donne une fausse impression de sécurité très dangereuse pour la constitution d'une théorie du langage qui aspire à la rigueur. L'utilité qu'on en attend se fonde sur de faux espoirs, des postulats incorrects. La recherche philosophique aurait beaucoup à gagner à se délivrer de ces mythes dépourvus de fondement théorique, même s'ils ont plusieurs usages pratiques. Les relations établies sont mal définies et ne nous font pas comprendre de manière fondée ce qu'est la signification.

Quine critique aussi la position du pragmatisme linguistique. Ce dernier a beau refuser, comme lui, la réification de la signification, il conduit à la postuler comme un modèle abstrait de l'usage des mots. Dès lors, il permet l'inférence de cette conclusion fallacieuse: si deux expressions linguistiques ont le même usage, elles sont synonymes. Ainsi, la position du pragmatisme linguistique permet de justifier théoriquement la relation de synonymie. Quine la refuse énergiquement. Il pense que l'observation de l'usage des mots peut justifier les interprétations les plus diverses. On n'est

7. Quine, W.V.O. Philosophy of Logic (Prentice Hall, 1970), p. 18. 
jamais en mesure d'inférer avec rigueur et univocité quelle est leur signification propre et, encore moins, si deux expressions ont ou n'ont pas la même signification. En outre, des hypothèses logiquement incompatibles seraient justifiées à partir des mêmes situations d'usage linguistique et on deviendrait incapable de décider laquelle serait correcte. La signification linguistique est sous-déterminée par toutes les observations possibles; elle ne se révèle pas empiriquement. Insistons sur cet aspect, car cette croyance constitue l'un des fondements de la thèse de l'indétermination de la traduction que nous examinons ici.

Faisons le point: à travers son analyse du processus d'apprentissage du langage, Quine conclut que la signification ne dépasse pas ce qu'on a assimilé quand on a appris à utiliser correctement les expressions linguistiques. Il s'agit du montage d'une série indéfinie de réflexes conditionnés qui mettent en jeu les organes qui nous permettent de parler, en réponse aux stimulations sensorielles. La signification se situe parmi les habitudes que nous acquérons et que rend possible la parole, en nous permettant de lier notre comportement verbal aux stimulations externes. À une étape plus complexe du processus d'apprentissage du langage - la phase d'inter-animation des énoncés - la signification dérive de l'ensemble des relations des énoncés entre eux. Quine aboutit à sa thèse du holisme sémantique.

\section{Le holisme sémantique}

Si l'analyse du processus d'apprentissage du langage révèle que la signification linguistique n'a pas de base empirique, elle ne saurait dériver que du réseau théorique auquel les énoncés appartiennent. Quine énonce ainsi les fondements de la thèse du holisme sémantique:

- l'unité de signification linguistique est la totalité de la théorie à laquelle un énoncé appartient, et jamais l'énoncé pris isolément;

- aucune expression, détachée de son contexte, ne possède une signification. 


\section{La thèse de l'inscrutabilité ${ }^{8}$ de la référence}

Toujours à partir de l'analyse du processus d'apprentissage du langage, Quine conclut que l'observation du comportement des locuteurs ne justifie pas plus la notion de signification que celle de référence.

Si les champs d'observation admettent plusieurs descriptions et si l'analyse des usages linguistiques se produit dans des cadres d'observation assez limités par rapport à tous les cas possibles, on ne pourra jamais tester toutes les hypothèses possibles.

L'analyse du processus d'apprentissage du langage révèle que les apprentis peuvent attribuer des significations et des références incompatibles aux mêmes expressions. Cela n'empêche pas que leurs usages s'accordent également bien à toutes les observations possibles. On ne sera jamais en mesure de décider, à partir de l'observation du comportement des locuteurs, quelles sont la signification et la référence correctes, puisque ces deux notions sont inscrutables du point de vue behavioriste. Donc, l'indétermination concerne non seulement la signification, mais la référence.

Quine aboutit à la constatation que la moindre unité référentielle du langage est l'ensemble théorique dont les énoncés sont partie intégrante. Détaché de cet ensemble, il est impossible d'assurer qu'un certain mot nomme un certain référent.

\section{La thèse de l'indétermination de la traduction}

Après avoir rappelé les postulats et l'articulation des arguments majeurs de la philosophie du langage de Quine, on est en mesure de comprendre ce qui fonde sa thèse de l'indétermination de la traduction.

Quine a ébauché pour la première fois les fondements de cette thèse dans son article «le Mythe de la signification», mais sa formulation précise et les arguments qui la soutiennent ne se trouvent clairement formulés que dans Word and Object.

En réponse aux innombrables critiques qu'elle a soulevées, Quine l'a reprise dans plusieurs articles comme: «Speaking of

8. Note de l'auteure: les lecteurs peu au fait des questions de philosophie analytique pourraient s'étonner de l'emploi en français d'une terminologie parfois empruntée à l'anglais. Des termes tels que «inscrutabilité», «inscrutable» ou encore «ostension» sont aujourd'hui couramment employés par celles et ceux qui travaillent sur ces questions. Il est par ailleurs à noter que les termes anglais originaux ne sont pas nécessairement plus usuels que ceux utilisés en français (cas de «ostension»), dans la mesure où ils relèvent de la terminologie de spécialité de Quine. 
Objects» (1958), «On the Reasons for Indeterminacy of Translation» (1970) et très récemment dans «Indeterminacy of Translation Again» (1987).

Son argumentation s'appuie sur les présupposés behavio-naturalistes de son analyse du langage et elle utilise une situation hypothétique bien particulière: la traduction radicale.

Le naturalisme l'amène à voir le discours comme un objet physique, qui a été produit en réponse à des stimulations physiques de nos terminaisons nerveuses et qui doit être analysé par des méthodes semblables à celles utilisées par les sciences de la nature, c'est-à-dire des méthodes empiriques.

Quant au choix d'une procédure behavioriste, elle l'a conduit à appuyer ses analyses sur l'observation du comportement des locuteurs, en évitant toutes solutions qui font appel à des entités mentales pour expliquer lés questions linguistiques.

Cette situation hypothétique est celle d'un linguiste qui envisage d'apprendre le langage et de construire un manuel de traduction des expressions linguistiques d'un peuple dont la langue et la culture sont totalement inconnues de lui. Il s'agit d'un peuple qui vit dans un état très primitif, sans aucune similitude directe de comportements avec ceux de la culture d'où provient le linguiste. Cela rend encore plus difficile ses analyses: il ne dispose ni d'interprètes, ni de dictionnaires; bref, il n'a comme point de repère que l'observation du comportement des locuteurs.

Ce linguiste sera donc obligé d'établir des hypothèses analytiques qui lui permettront d'aboutir aux parallélismes entre la signification des expressions de la langue de ce peuple et celles de sa langue maternelle.

Il sera obligé d'établir certaines hypothèses; par exemple:

- empathie: le linguiste doit éviter des résultats qui impliquent que ce peuple puisse avoir des croyances très aberrantes par rapport aux siennes;

- continuité des croyances: le manuel de traduction qu'il produit ne peut pas nous faire penser que ce peuple change de croyances tout le temps;

- simplicité: le manuel doit révéler une structure linguistique qui ne soit pas trop compliquée.

Le degré d'efficacité du manuel est fonction de la réussite ou de l'échec du dialogue du linguiste avec son interlocuteur. Ce sera par conséquent dans un cadre pragmatique qu'on pourra vérifier la validité des hypothèses choisies.

La situation qui est prise à titre d'exemple est celle où le linguiste entend l'expression «gavagai» et s'efforce de la traduire. 
En connaissant déjà les expressions qui expriment l'affirmation et la négation dans ce langage, il reproduit une série de situations expérimentales destinées à appréhender la signification de «gavagai». Il constate que cette expression s'applique à des situations qui concernent les lapins. Mais aucune observation expérimentale ne lui permettra de décider si elle correspond, par exemple, à l'expression «lapin», «lapinité» ou «parties non détachées de lapin». Toutes les expériences possibles justifieraient également bien le choix d'une ou l'autre de ces expressions.

Quand le linguiste décide de traduire «gavagai» par «lapin», il le fait en transcendant les données d'observation en fonction d'hypothèses analytiques qu'il a construites, qui dépendent fondamentalement de sa théorie du monde. Le choix entre les expressions «lapin», «lapinité», «parties non détachées de lapin», comme traduction de "gavagai», dépend de théories hypothétiques du monde, lesquelles sont logiquement incompatibles. Ces expressions diffèrent en ce qui concerne non seulement leur «intension», mais leur «extension»: elles sont vraies de choses différentes. Et aucun critère de décision ne sera capable de justifier l'affirmation qu'il y a une meilleure option parmi les trois.

Quine est convaincu que, si deux linguistes entreprennent cette tâche et s'ils n'entrent pas en contact l'un avec l'autre, ils produiront des manuels de traduction différents, incompatibles du point de vue logique. Néanmoins, leurs deux manuels seront également compatibles avec le comportement du peuple observé.

Ainsi, il peut énoncer sa thèse:

Si la signification et la référence sont behavioristiquement inscrutables, il est possible de construire des manuels de traduction logiquement incompatibles et empiriquement équivalents. Il est impossible d'arriver à des critères assez rigoureux pour permettre de justifier la conclusion qu'un manuel donné est le seul correct. On pourra certes, à la limite, disqualifier ceux qui produisent des traductions trop incorrectes, mais en cas de conflit entre deux traductions bien appuyées sur des données d'observation, on ne sait plus comment décider laquelle est correcte.

Quine conclut que toutes les traductions sont indéterminées par rapport à l'observation du comportement linguistique, y compris celles des énoncés d'observation qui sont cependant les moins susceptibles d'indétermination.

Ainsi, traduire consiste à établir des hypothèses analytiques à partir de données observées. Le traducteur imposera son système référentiel et logique, ses modèles ontologiques et culturels pour établir des corrélations sémantiques entre les deux langues. 
Cette thèse n'a rien de nihiliste. Quine soutient que les traductions sont indispensables. Il est indiscutable qu'on traduit constamment et on le fait tant au niveau intra-linguistique qu'inter-linguistique. "L'indétermination commence chez nous», affirme Quine. Cela veut dire que même à l'intérieur de notre communauté linguistique, il n'y a pas de moyen de déterminer de façon univoque les significations des expressions à partir de l'observation du comportement des locuteurs. Ainsi, les rapports intralinguistiques, en ce qui concerne les significations, ont lieu aussi dans le cadre des hypothèses analytiques que chaque locuteur produit et des accords pragmatiques auxquels on aboutit à partir de là.

Il est très important de souligner que, contrairement à ce qu'affirment nombre des critiques qui n'ont pas compris cette thèse, Quine n'a jamais dit qu'il est impossible de traduire, ou qu'il n'y a pas de traduction acceptable. Ce qu'il soutient en revanche, c'est qu'il y aura toujours plusieurs traductions possibles, toutes avec un très haut degré d'acceptabilité quand elles sont d'accord avec les données d'observation sur lesquelles on s'est appuyé pour construire les hypothèses analytiques.

Il y a un profond rapport entre les observations du comportement verbal d'un peuple et les traductions de ses structures linguistiques. Le problème théorique qui se pose, c'est que ce rapport ne suffit pas à produire un seul manuel de traduction, puisque la signification linguistique est insuffisamment déterminée par toutes les observations possibles du comportement linguistique. Dès qu'il y a désaccord sur la signification d'une expression, répétons-le, on ne dispose d'aucun moyen de décision qui nous permette d'inférer avec rigueur qu'elle est la seule expression correcte.

On se rend compte que l'argumentation quinéenne ne l'amène pas à la conclusion qu'il est impossible de traduire, mais plutôt qu'il y a une infinité de traductions possibles, toutes logiquement incompatibles mais empiriquement équivalentes.

L'attitude quinéenne relativement à la sémantique n'est pas la répudiation ou la dévalorisation. Il entend stimuler son développement, d'après la démarche des sciences naturelles, ce qui confère à ses analyses la rigueur scientifique souhaitable. La priorité doit être accordée aux théories appuyées sur les mécanismes physiques observables, à l'exclusion des explications mentalistes, internalistes.

Les recherches dans le domaine des sciences du langage auront, semble-t-il, beaucoup à gagner en s'associant aux analyses des sciences de la nature, notamment à la neurophysiologie. 


\section{La thèse Duhem-Quine et le holisme épistémologique}

Il faut également faire mention des critiques adressées à Quine. Certains, comme N. Chomsky, affirment que la thèse de l'indétermination de la traduction est un cas particulier de sa thèse de la sous-détermination des théories par les événements, ce qui la rendrait triviale. Pour répondre à ces objections, il importe d'abord de présenter la thèse.

Quine est d'accord avec Duhem sur le fait que les théories physiques sont sous-déterminées par toutes les diverses observations possibles. Ainsi, il accepte les postulats suivants de Duhem:

- les théories physiques dépassent toutes les observations possibles du monde;

- des théories diverses et en conflit peuvent s'appuyer sur les mêmes données d'observation.

Voici la thèse: il est toujours possible de construire des théories logiquement incompatibles et empiriquement équivalentes.

C'est une thèse plus étendue que celle de Duhem qui l'appliquait seulement à l'indétermination des théories physiques. Quine émet l'hypothèse que toutes les théories du monde - scientifiques, philosophiques ou du sens commun - seront toujours sousdéterminées par l'expérience. La conséquence qui en dérive, selon lui, c'est que la signification, la référence et la valeur de vérité de chaque énoncé dépendent de la façon dont l'ensemble théorique entre en rapport avec les événements décrits, ce qui justifie son holisme épistémologique:

Nos énoncés sur le monde extérieur affrontent le tribunal de

l'expérience sensible, non individuellement, mais comme un tout bien structuré. 9

Relativement à son projet épistémologique, il constate la faiblesse de la méthode behavioriste qu'il a utilisée dans son analyse du langage - la seule qu'il estime valable. Mais sa méthode lui a permis d'expliquer comment lier la production de nos théories du monde aux stimulations de nos terminaisons nerveuses - qui demeure sa principale préoccupation épistémologique. En même temps, elle lui a fait comprendre que, à cause de la phase d'inter-animation des énoncés, les théories du monde dépassent largement les données de l'observation et qu'elles dépendent, dans une large mesure, d'un contexte théorique plus vaste. Ainsi, les observations - scientifiques ou du sens commun - pourront

9. Quine, W.V.O. Methods of Logic (Routledge, 1974), p. 12. 
refléter toujours le cadre théorique dans lequel elles ont lieu. Cela nous empêche de distinguer avec rigueur dans notre discours les énoncés qui répondent directement aux stimulations physiques et ceux qui proviennent des rapports avec l'ensemble théorique par inter-animation.

L'accord entre les théories et les données empiriques est toujours global; il n'est jamais atomisé. Au cas où elles ne s'accorderaient pas, $c$ 'est la théorie qui devra être globalement révisée et cette révision doit s'appliquer à tous les énoncés, y compris les énoncés mathématiques et les énoncés éternels (ceux qui sont vrais ou faux indépendamment des circonstances de leur utilisation). Les principes de simplicité, de conservation sont des critères de décision pour choisir ce qui doit être conservé et ce qui doit être renouvelé dans l'ensemble considéré.

La sous-détermination des théories diffère de l'indétermination des traductions en raison d'un aspect fondamental: l'indétermination concerne l'impossibilité où se trouve la signification de se manifester empiriquement, puisqu'elle n'est pas une entité. Rien dans les données de l'observation ne pourra justifier l'option pour tel ou tel manuel. Aucune situation, même hypothétique, ne permettra de sortir d'une telle impasse: différents usages linguistiques pourront justifier de la même manière des hypothèses analytiques en conflit. En revanche, dans le cas des théories, on pourra toujours penser à des expériences possibles, même si elles sont irréalisables, susceptibles de résoudre le problème de la sous-détermination (puisqu'elles ont affaire à des objets physiques ou théoriques).

Quine conclut: à supposer qu'on puisse trouver des solutions à toutes les sous-déterminations dans le domaine des théories scientifiques, cela n'amènerait pas à éliminer l'indétermination des traductions. Il ne s'agit pas d'un cas particulier de l'ensemble des sous-déterminations des théories; il s'agit de tout autre chose. On y est confronté toutes les fois que l'on essaie de traduire des discours scientifiques d'un langage à un autre ou quand on envisage de faire des traductions intra-théoriques.

\section{Conclusion}

Comme nous l'annoncions en commençant, l'objectif de cet essai est de situer la thèse de l'indétermination de la traduction dans l'ensemble du système philosophique de Quine. Nous croyons que c'est la seule façon d'éviter un grand nombre d'équivoques.

Pour cela, nous avons voulu montrer les rapports qu'elle entretient avec son projet épistémologique, avec la théorie behavio-naturaliste de l'apprentissage du langage, avec la critique du 
mythe de la signification et du manque de rigueur de la notion de synonymie, avec le holisme sémantique et épistémologique, et enfin avec l'inscrutabilité de la référence et la thèse Duhem-Quine.

Cette analyse révèle que la thèse de l'indétermination de la traduction est parfaitement cohérente avec l'ensemble de la philosophie du langage quinéenne et que les arguments qui la soutiennent sont solides dès qu'on accepte les postulats de l'argumentation.

Nous croyons aussi que cette thèse se maintient même si on refuse les postulats behavioristes. Ce qui la soutient fondamentalement, c'est l'absence de "fact of matter», sur lequel s'appuyer objectivement pour décider entre deux possibilités logiquement incompatibles, mais empiriquement équivalentes, de traduire une certaine expression linguistique. Il ne semble pas qu'on puisse trouver des contre-exemples à cette affirmation de Quine.

Une bonne méthode pour tester la thèse de l'indétermination de la traduction consiste à la faire entrer en dialogue avec ses critiques: ce faisant, on constate qu'on est impuissant à la démolir.

Nous sommes d'accord avec ceux qui disent que si l'on suivait les principes de la sémantique quinéenne on ne serait pas en mesure d'avancer beaucoup. Peut-être faut-il reconnaître, en effet, que les instruments quinéens ont beau être cohérents, ils ne sont pas forcément suffisants pour relever les défis qui se présentent à la sémantique contemporaine. Les postulats de sa philosophie du langage ne permettent pas toujours de résoudre les impasses avec lesquelles les analystes du langage sont aux prises. Cela ne suffit pas pour autant à invalider la force et la cohérence de ses thèses.

Université fédérale de Rio de Janeiro 


\section{Références}

FrIEDMAN, M. (1975). «Physicalism and the Indeterminacy of Translation», Nous.

Quine, W.V.O. (1969). Ontological Relativity and Other Essays, New York, Columbia University Press. (1960). Word and Object, Cambridge, M.I.T. Press. (1970). Philosophy of Logic. Prentice Hall. (1981). Theories and Things. Harvard University Press. (1987). «Indeterminacy of Translation Again». The Journal of Philosophy, LXXXIV(1).

(1975). «The Nature of Natural Knowledge». Mind and Language, éd. S. Gưttenplan, Oxford, Clarendon. (1975). «Linguistics and Philosophy». Mind and Language, éd. S. Guttenplan, Oxford, Clarendon. (1962). «Le mythe de la signification». La Philosophie analytique, Paris, Éd. de Minuit. (1974). The Roots of Reference. La Salle, Open Court. (1958). «Speaking of Objects». Ontological Relativity and Other Essays. New York, Columbia U.P. (1970). «On the Reasons for Indeterminacy of Translation». The Journal of Philosophy, LXVII(6). 